\title{
BIBLIOGRAPHY
}

1. K. L. Chung, Fluctuation of sums of independent random variables, Ann. of Math. vol. 51 (1950) pp. 697-706.

2. K. L. Chung and P. Erdös, Probability limit theorems assuming only the first moment. I, Memoirs of the American Mathematical Society, no. 6, pp. 13-19.

3. - - On the lower limit of sums of independent random variables, Ann. of Math. vol. 48 (1947) pp. 1003-1013.

4. K. L. Chung and W. H. J. Fuchs, On the distribution of values of sums of random variables, Memoirs of the American Mathematical Society, no. 6, pp. 1-9.

5. H. Cramér, Random variables and probability distributions, Cambridge Tracts in Mathematics, no. 36, 1937.

6. C. G. Esseen, Fourier analysis of distribution functions, Acta Math. vol. 77 (1945) pp. 5-145.

7. G. Pblya, Über den zentralen Grenzwertsatz der Wahrscheinlichkeitsrechnung und das Momentproblem, Math. Zeit. vol. 8 (1920) pp. 171-181.

8. G. H. Hardy and E. M. Wright, An introduction to the theory of numbers, Oxford, 1938.

Columbia University

\section{ON PROJECTIVE GEOMETRY OVER FULL MATRIX RINGS}

RIMHAK REE

1. Introduction. In this note we show that projective geometry over a ring $R$ and that over the full matrix ring $R_{n}$ are essentially the same, and extend the fundamental theorem of projective geometry $[1$, p. 44$]$ to the case of $\Phi_{n}$-modules, where $\Phi$ is a division ring. (By a projective geometry over $R$ we mean a lattice of all $R$-submodules of an $R$-module.) As a special case of these results we have the following: If $n \geqq 3$, any lattice isomorphism of the lattice of all left ideals of $\Phi_{n}$ and that of $\Psi_{m}$ where $\Phi$ and $\Psi$ are division rings, is induced by an isomorphism of $\Phi_{n}$ and $\Psi_{m}$. We obtain also an extension of the basis theorem for vector spaces to $\Phi_{n}$-modules.

Other extensions of the fundamental theorem of projective geometry have been made by Baer, for the case of $R$-modules, where $R$ is a "primary ring" in his sense [2, p. 304], and the ring of rational integers [3].

2. Main theorems. In the following, by a ring we always mean an associative ring with unit element. Let $R$ be a ring with unit

Received by the editors April 17, 1954. 
element 1 . An additive group $A$ is called an $R$-module if $a x$ is defined for all $a$ in $R$ and all $x$ in $A$ such that

$$
\begin{aligned}
a(x+y) & =a x+a y, \\
(a+b) x & =a x+b x, \\
a(b x) & =(a b) x, \\
1 x & =x
\end{aligned}
$$

for any $a, b \in R$ and any $x, y \in A$. A subgroup $B$ of $A$ is called an $R$-submodule if $a x \in B$ for all $a \in R$ and all $x \in B$. The set of all $R$-submodules of $A$ forms a modular lattice $L(R, A)$ with respect to grouptheoretical union and intersection. We will call the lattice $L(R, A)$ a projective geometry over the ring $R$. (Usual projective geometry is the case when $R$ is a division ring.)

We denote by $R_{n}$ the full matrix ring of degree $n$ over $R$. Our first main theorem is the following:

THEOREM 1. Let $R$ be a ring and $n$ be a positive integer. Then for any $R_{n}$-module $M$ there exists an $R$-module $A$ such that

$$
L\left(R_{n}, M\right) \cong L(R, A) .
$$

Conversely, for any $R$-module $A$ there exists an $R_{n}$-module $M$ such that (1) holds.

Now let $\mathfrak{F}=\{R, S, \cdots\}$ be a family of rings and $\pi$ be a lattice theoretical condition. We shall say that the fundamental theorem of projective geometry (f.t.p.g.) holds in $\mathfrak{F}$ under $\pi$ if, for any ring $R$ in $\mathfrak{F}$ and $R$-module $A$ such that $L(R, A)$ satisfies the condition $\pi$, the following holds:

(2) If $S$ is a ring in $\mathfrak{F}$ and if $B$ is an $S$-module such that $L(R, A)$ $\cong L(S, B)$, then there exists an isomorphism $\sigma$ of $A$ to $B$ and an isomorphism $\sigma^{\prime}$ of $R$ to $S$ such that the lattice isomorphism $A_{1} \rightarrow A_{1}^{*}$ $=B_{1}$, where $A_{1}$ denotes an arbitrary element in $L(R, A)$, is induced by $\sigma$, i.e.

$$
A_{1}^{*}=\left\{y^{\sigma} \mid y \in A_{1}\right\},
$$

and such that $(a x)^{\sigma}=a^{\sigma \prime} x^{\sigma}$ for all $a$ in $R$ and all $x$ in $A$. Our second main theorem is the following:

TheOREM 2. Let $\mathfrak{F}=\{R, S, \cdots\}$ be a family of rings, $n$ a positive integer, and $\pi$ be a lattice-theoretic condition. If f.t.p.g. holds in $\mathfrak{F}$ under $\pi$ then f.t.p.g. also holds in $\mathfrak{F}_{n}=\left\{R_{n}, S_{n}, \cdots\right\}$ under $\pi$. The isomorphism $\sigma^{\prime}$ of $R_{n}$ to $S_{n}$ needed in f.t.p.g. in $\mathfrak{F}_{n}$ can always be chosen so 
that $\sigma^{\prime}$ is of the type $\left(a_{i j}\right)^{\sigma^{\prime}}=\left(a_{i j}^{\sigma \prime \prime}\right)$, where $\sigma^{\prime \prime}$ is a suitable isomorphism of $R$ to $S$.

3. Proof of Theorem 1. Let $e_{i j}$ be the $n \times n$ matrix with 1 in the $i$ th row and $j$ th column and 0 elsewhere. It is easily seen that $e_{i i}$ commutes with any diagonal matrix. Let $a$ be an element in $R$. We shall denote by $[a]$ the diagonal matrix of which all the diagonal elements are equal to $a$. The set of all [a] forms a subring of $R_{n}$ isomorphic to $R$.

Let $M$ be a given $R_{n}$-module. If we set $A=e_{11} M$, then we can consider $A$ as an $R$-module, since every $[a]$ is commutative with $e_{11}$. If $M_{1}$ is an $R_{n}$-submodule of $M$, then $A_{1}=e_{11} M_{1}$ is an $R$-submodule of $A$. We shall show that the mapping $\phi: M_{1} \rightarrow A_{1}=e_{11} M_{1}$ gives the desired isomorphism of $L\left(R_{n}, M\right)$ and $L(R, A)$. Let $M_{1}, M_{2}$ be two $R_{n}$-submodules of $M$ such that $e_{11} M_{1} \subseteq e_{11} M_{2}$. We shall show that $M_{1} \subseteq M_{2}$. For let $x \in M_{1}$. Then $e_{1 i} x \in M_{1}$, and $e_{1 i} x=e_{11}\left(e_{1 i} x\right) \in e_{11} M_{1}$ $\subseteq e_{11} M_{2} \subseteq M_{2}$. Thus $e_{1 i} x \in M_{2}$, and $e_{i i} x=e_{i 1}\left(e_{1 i} x\right) \in e_{i 1} M_{2} \subseteq M_{2}$. Hence $e_{i i} x \in M_{2}$ for $i=1, \cdots, n$, and since $e_{11}+\cdots+e_{n n}$ is the unit element of $R_{n}, x \in M_{2}$. Thus $M_{1} \subseteq M_{2}$ is proved. If $e_{11} M_{1}=e_{11} M_{2}$ then clearly $M_{1}=M_{2}$. Hence we have proved that $\phi$ is univalent and preserves inclusion. Let $A_{1}$ be any $R$-submodule of $A$, and let $M_{1}$ be the set of all elements $x$ in $M$ such that $e_{1 i} x \in A_{1}$ for all $i=1, \cdots, n$. Then $M_{1}$ is an $R_{n}$-submodule of $M$, for if $x \in M_{1}$ then $e_{i j} x \in M_{1}$ for $i, j=1, \cdots, n$ and $[a] x \in M_{1}$ for all $a$ in $R$, since $e_{1 k}\left(e_{i j} x\right)=\delta_{k i} e_{1 j} x \in A_{1}$, where $\delta_{k i}$ is the Kronecker delta. Since $[a]$ and $e_{1 i}$ permute, and $A_{1}$ is an $R$-submodule of $A,[a] x \in M_{1}$, so that $M_{1}$ is proved to be an $R_{n}$-submodule of $M$. We shall show that $A_{1}=e_{11} M_{1}$. Since $e_{11} x \in A_{1}$ for all elements $x$ of $M_{1}$, we have $e_{11} M_{1} \subseteq A_{1}$. Let $x \in A_{1}$; then since $A_{1} \subseteq A=e_{11} M, x=e_{11} x^{\prime}$ for some $x^{\prime} \in M$, and $e_{11} x=x \in A_{1}, e_{1 i} x=e_{1 i}\left(e_{11} x\right)$ $=0$ for $i>1$. Therefore $x \in M_{1}$, and $x=e_{11} x \in e_{11} M_{1}$. Thus $A_{1}=e_{11} M_{1}$ is proved.

To prove the second part of Theorem 1 let $A$ be a given $R$-module. Let $M$ be the totality of $n$-uples $\left(x_{1}, \cdots, x_{n}\right)$ of elements of $A$. If we define addition in $M$ by adding component-wise, $M$ becomes an additive group (direct sum of $n$ copies of $A$ ). Let $\left(a_{i j}\right)$ be an arbitrary element of $R_{n}$, and define $\left(a_{i j}\right)\left(x_{1}, \cdots, x_{n}\right)=\left(y_{1}, \cdots, y_{n}\right)$ by $y_{i}$ $=a_{i 1} x_{1}+\cdots+a_{i n} x_{n}$ for $i=1, \cdots, n$. Then $M$ becomes an $R_{n}$-module, and the $R$-module $e_{11} M$ is clearly isomorphic to the $R$-module $A$. That $L\left(R_{n}, M\right) \cong L(R, A)$ follows as in the proof of the first half of Theorem 1 , so that Theorem 1 is completely proved.

4. Proof of Theorem 2. Suppose that the f.t.p.g. holds in $\mathfrak{F}=\{R, S, \cdots\}$ under $\pi$, that $M$ is an $R_{n}$-module, and that $L\left(R_{n}, M\right)$ 
satisfies $\pi$. Let $M_{1} \rightarrow M_{1}^{*}=N_{1}$ be a lattice isomorphism of $L\left(R_{n}, M\right)$ and $L\left(S_{n}, N\right)$, where $N$ is an $S_{n}$-module. By Theorem 1 there exists an $R$-module $A$ and $S$-module $B$ such that $L\left(R_{n}, M\right) \cong L(R, A)$, $L\left(S_{n}, N\right) \cong L(S, B)$. Then $L(R, A) \cong L(S, B)$ and clearly both of these lattices satisfy $\pi$. In view of the proof of Theorem 1, we may assume that $A=e_{11} M, B=e_{11} N$. Now by our assumption there is an isomorphism $\sigma$ of $e_{11} M$ to $e_{11} N$ and an isomorphism $\sigma^{\prime}$ of $R$ to $S$ such that $e_{11} M_{1}^{*}=\left\{y^{\sigma} \mid y \in e_{11} M_{1}\right\}$ for every $R_{n}$-submodule $M_{1}$ of $M$, and such that $\left([a] e_{11} x\right)^{\sigma}=\left[a^{\sigma \prime}\right]\left(e_{11} x\right)^{\sigma}$ for every $a$ in $R$ and every $x$ in $M$. Now define a mapping $\tau: x \rightarrow x^{\tau}$ from $M$ to $N$ by

$$
x^{r}=e_{11}\left(e_{11} x\right)^{\sigma}+e_{21}\left(e_{12} x\right)^{\sigma}+\cdots+e_{n 1}\left(e_{1 n} x\right)^{\sigma} .
$$

Note that $\left(e_{1 i} x\right)^{\sigma}$ is meaningful for $x \in M$ and $i=1, \cdots, n$, since $e_{1 i} x=e_{11}\left(e_{1 i} x\right) \in e_{11} M$. We shall show that $\tau$ is an isomorphism of $M$ and $N$. Since $\sigma$ is an isomorphism of $e_{11} M$ to $e_{11} N$, we have easily $(x+y)^{\tau}=x^{\tau}+y^{\tau}$. If $x^{\tau}=0$, then $e_{1 i} x^{\tau}=0$ and therefore $e_{1 i} e_{i 1}\left(e_{13} x\right)^{\sigma}$ $=e_{11}\left(e_{1 i} x\right)^{\sigma}=0$. Since $\left(e_{1 i} x\right)^{\sigma} \in e_{11} N$ we have $\left(e_{1 i} x\right)^{\sigma}=e_{11}\left(e_{1 i} x\right)^{\sigma}=0$. Hence $e_{1 i} x=0$, since $\sigma$ is an isomorphism. Then $e_{i i} x=e_{i 1} e_{1 i} x=0$ for $i=1, \cdots, n$. Therefore $x=0$. Now we have to show that for any $z \in N$ there is an $x \in M$ such that $z=x^{\tau}$. Since $e_{1 i} z=e_{11}\left(e_{1 i} z\right) \in e_{11} N$ there exists an element $x_{i}$ in $e_{11} M$ such that $e_{1 i} z=x_{i}^{\sigma}$. Put $x=e_{11} x_{1}+e_{21} x_{2}+\cdots+e_{n 1} x_{n}$. Then $e_{1 i} x=e_{11} x_{i}=x_{i}$. Hence $e_{1 i} z=\left(e_{1 i} x\right)^{\sigma}$, and $e_{i i} z=e_{i 1}\left(e_{1 i} x\right)^{\sigma}$. Therefore $z=x^{\tau}$. Thus we have proved that $\tau$ is an isomorphism of $M$ to $N$. Now for any $a \in R$ and $x \in M$ we have

$$
\begin{aligned}
([a] x)^{r} & =\sum e_{i 1}\left(e_{1 i}[a] x\right)^{\sigma}=\sum e_{i 1}\left([a] e_{1 i} x\right)^{\sigma} \\
& =\sum e_{i 1}\left[a^{\sigma^{\prime}}\right]\left(e_{1 i} x\right)^{\sigma}=\left[a^{\sigma^{\prime}}\right] \sum e_{i 1}\left(e_{1 i} x\right)^{\sigma} \\
& =\left[a^{\sigma^{\prime}}\right] x^{\tau}
\end{aligned}
$$

and $\left(e_{i j} x\right)^{\tau}=e_{i 1}\left(e_{1 j} x\right)^{\sigma}=e_{i j} x^{\tau}$. Therefore for any $\left(a_{i j}\right) \in R_{n}$ and $x \in M$ we have $\left(\left(a_{i j}\right) x\right)^{\tau}=\left(a_{i j}^{\sigma^{\prime}}\right) x^{\tau}$. Finally we have to show that $M_{1}^{*}$ $=\left\{x^{r} \mid x \in M_{1}\right\}$ for an arbitrary $R_{n}$-submodule $M_{1}$ of $M$. We note that $y^{\tau}=y^{\sigma}$ for all $y \in e_{11} M$. Let $x$ be in $M_{1}$. Since we have $e_{11} M_{1}^{*}$ $=\left\{y^{\sigma} \mid y \in e_{11} M_{1}\right\}$, and since $e_{1 i} x \in e_{11} M_{1}$, we have $e_{1 i} x^{r}=\left(e_{1 i} x\right)^{r}$ $=\left(e_{1 i} x\right)^{\sigma} \in e_{11} M_{1}^{*}$. Hence $e_{i i} x^{\tau} \in e_{i 1} M_{1}^{*} \subseteq M_{1}^{*}$. Therefore $x^{\tau} \in M_{1}^{*}$. Conversely, let $z$ be in $M_{1}^{*}$. Then we can find $x_{i}$ in $e_{11} M_{1}$ such that $e_{1 i} z$ $=x_{i}^{\sigma}=x_{i}^{\tau}$. Hence $e_{i i} z=e_{i 1} x_{i}^{\tau}=\left(e_{i 1} x_{i}\right)^{r}$ and $z=\sum\left(e_{i 1} x_{i}\right)^{r}=\left(\sum e_{i 1} x_{i}\right)^{\tau}$. Clearly $x=\sum e_{i 1} x_{i}$ is in $M_{1}$, and $z=x^{\tau}$. Thus we have proved that the lattice isomorphism of $L\left(R_{n}, M\right)$ to $L\left(S_{n}, N\right)$ is induced by the groupisomorphism $\tau$. Theorem 2 is completely proved.

5. Applications. Theorem 1 shows that for any division ring $\Phi$ and any $\Phi_{n}$-module $M$ the lattice $L\left(\Phi_{n}, M\right)$ is complementary. 
To move from a projective geometry over $\Phi$ to one over $\Phi_{n}$ more effectively, we need lattice-theoretic definitions of a few concepts in vector spaces. Let $L$ be a lattice with join $U$ and meet $\cap$. We shall write $X \leqq Y$ if $X \cap Y=X$. Assume that $L$ has a smallest element 0 , that is, 0 is an element such that $0 \leqq X$ for all $X \in L$. A finite set $X_{1}, \cdots, X_{r}$ of elements $\neq 0$ in $L$ are said to be independent if $X_{i} \cap\left(X_{1} \cup \ldots \cup X_{i-1} \cup X_{i+1} \cup \ldots \cup X_{r}\right)=0$ for $i=1, \cdots, r$. A set $\left\{X_{\alpha}\right\}$ of elements are said to be independent if every finite subset of $\left\{X_{\alpha}\right\}$ is independent. An element $X \neq 0$ in $L$ is said to be minimal if $X \geqq Y>0$ implies $X=Y$. An independent set $\mathfrak{B}=\left\{X_{\alpha}\right\}$ of minimal elements is called a basis of $L$ if for every minimal element $X$ there exist finite number of elements $X_{1}, \cdots, X_{n}$ in $\mathfrak{B}$ such that $X$ $\leqq X_{1} \cup \ldots \cup X_{n}$. The well-known basis theorem of vector spaces $[1$, p. 14] can easily be seen to be equivalent to the following: If $\Phi$ is a division ring then for any $\Phi$-module $A$ the lattice $L(\Phi, A)$ has a basis, and any two bases of $L(\Phi, A)$ have the same (cardinal) number of elements. Now Theorem 1 shows that for any $\Phi_{n}$-module $M$ the lattice $L\left(\Phi_{n}, M\right)$ has a basis, and that any two bases of $L\left(\Phi_{n}, M\right)$ have the same number of elements. From this we may readily prove the following extension of the basis theorem of vector spaces:

Let $\Phi$ be a division ring, $n$ be a positive integer, and $M$ be a $\Phi_{n}$-module. Then there exists a set $\mathfrak{B}=\left\{x_{\alpha}\right\}$ of elements in $M$ such that

(i) $\Phi_{n} x_{\alpha}$ is isomorphic to the $n$-dimensional vector space over $\Phi$ for any $x_{\alpha}$ in $\mathfrak{B}$,

(ii) any element $x$ in $M$ can be expressed as a linear combination $x=a_{1} x_{\alpha_{1}}+\cdots+a_{r} x_{\alpha_{r}}$ with $x_{\alpha_{1}}, \cdots, x_{\alpha_{r}}$ in $\mathfrak{B}$ and $a_{1}, \cdots, a_{r}$ in $\Phi_{n}$,

(iii) $a_{1} x_{\alpha_{1}}+\cdots+a_{r} x_{\alpha_{r}}=0$ with $x_{\alpha_{1}}, \cdots, x_{\alpha_{r}}$ in $\mathfrak{B}$ and $a_{1}, \cdots, a_{r}$ in $\Phi_{n}$ implies $a_{1} x_{\alpha_{1}}=\cdots=a_{r} x_{\alpha_{r}}=0$. Any two sets $\mathfrak{B}, \mathfrak{B}^{\prime}$ satisfying (i)-(iii) have the same number of elements.

Theorem 2 gives us at once an extension of f.t.p.g. to the case of simple rings $\Phi_{n}$, since for any family $\mathfrak{F}=\{\Phi, \Psi, \cdots\}$ of division rings f.t.p.g. holds under the condition $\pi$ : "there are at least three independent elements" [1, p. 44]. A similar extension when $R$ is the ring of all integers, if we use a result of Baer [3, p. 39]: If the abelian group $G$ contains at least two independent elements of infinite order, and if $H$ is an abelian group such that the lattice of all subgroups of $G$ is isomorphic to that of $H$, then the lattice isomorphism is induced by an isomorphism of $G$ and $H$. In this case we have to consider only torsion free modules.

Now let $R$ be a ring and $A=R \oplus \cdots \oplus R$ be a direct sum of $n$ copies of the additive group of $R$. If we define $a x=\left(a a_{1}, \cdots, a a_{n}\right)$ for $a \in R$ and $x=\left(a_{1}, \cdots, a_{n}\right) \in A$, then $A$ becomes an $R$-module. 
We assume that $R$ satisfies the following two conditions:

(i) Any lattice automorphism of $L(R, A)$ is induced by some automorphism $\sigma$ of $A$ such that $(a x)^{\sigma}=a^{\sigma^{\prime}} x^{\sigma}$ for every $a \in R, x \in A$, where $\sigma^{\prime}$ is an automorphism of $R$.

(ii) Let $P, Q$ be in the full matrix ring $R_{n}$. If $P Q=1$ then $Q P=1$. $Q$ will be denoted by $P^{-1}$.

THEOREM 3. Let a ring $R$ and an integer $n$ be such that the above two conditions (i), (ii) are satisfied. Then any automorphism $\alpha$ of the full matrix ring $R_{n}$ is of the form:

$$
\left(a_{i j}\right)^{\alpha}=U^{-1}\left(a_{i j}^{\alpha^{\prime}}\right) U
$$

where $\alpha^{\prime}$ is an automorphism of $R$, and $U$ is an element of $R_{n}$ for which $U^{-1}$ exists.

Proof. We consider $R_{n}$ as an $R_{n}$-module. Then $L\left(R_{n}, R_{n}\right)$ is the lattice of all left ideals of $R_{n}$. Let $\mathfrak{l}$ be an arbitrary left ideal of $R_{n}$. If we set $\mathfrak{l}^{\alpha}=\left\{\left(a_{i j}\right)^{\alpha} \mid\left(a_{i j}\right) \in \mathfrak{l}\right\}$, then $\alpha$ becomes a lattice-automorphism of $L\left(R_{n}, R_{n}\right)$. Now in view of the proof of Theorem 1 , we know that $L\left(R_{n}, R_{n}\right) \cong L(R, A)$. Therefore there is a lattice-automorphism $\beta$ of $L(R, A)$ corresponding to the automorphism $\alpha$ of $L\left(R_{n}, R_{n}\right)$. By our assumption $\beta$ is induced by an automorphism $\sigma$ of $A$ such that $(a x)^{\sigma}=a^{\sigma^{\prime}} x^{\sigma}$ for $a \in R, x \in A$, where $\sigma^{\prime}$ is an automorphism of $R$. Now in view of the proof of the second part of Theorem 2 there is an automorphism $\tau$ of the additive group of $R_{n}$ such that $\tau$ induces the same automorphism of $L\left(R_{n}, R_{n}\right)$ as $\alpha$ and such that $\left(\left(a_{i j}\right)\left(b_{i j}\right)\right)^{r}=\left(a_{i j}^{\sigma \prime}\right)\left(b_{i j}\right)^{r}$ for any $\left(a_{i j}\right),\left(b_{i j}\right) \in R_{n}$. We set [1] $=V$, then for any $\left(a_{i j}\right) \in R_{n}$ we have $\left(a_{i j}\right)^{r}=\left(a_{i j}^{\sigma \prime}\right) V$. Since $\tau$ is an automorphism of the additive group of $R_{n}$, there exists an element $\left(b_{i j}\right)$ such that $\left(b_{i j}\right)^{r}=1$, so that if we set $W=\left(b_{i j}^{\sigma \prime}\right)$ then $1=W V$. Now by our assumption [1] $=V W$. Therefore if we set $\left(a_{i j}\right)^{\eta}=W\left(a_{i j}^{\sigma \prime}\right) V$ then $\eta$ is an automorphism of $R_{n}$. It is easily seen that $\eta$ induces the same lattice automorphism of $L\left(R_{n}, R_{n}\right)$ as $\tau$, and consequently, as $\alpha$.

Now we set $\iota=\alpha \eta^{-1}$. Then $\iota$ is an automorphism of $R_{n}$, which induces the identity lattice automorphism of $L\left(R_{n}, R_{n}\right)$. We set

$$
P=\sum_{i=1}^{n} e_{i 1}^{\imath} e_{1 i}, \quad Q=\sum_{i=1}^{n} e_{i 1}^{\imath} e_{1 i} .
$$

Then $P Q=\sum e_{11}^{\imath} e_{11} e_{11}^{\imath}$. Since the left ideal $R_{n} e_{11}$ is invariant under the automorphism $\iota$, we have $e_{i 1}^{\imath}=S_{i} e_{i 1}$ for some $S_{i} \in R_{n}$. Then $e_{i 1}^{\imath} e_{11}$ $=S_{i} e_{i 1} e_{11}=S_{i} e_{i 1}=e_{i 1}^{\imath}$. Therefore $P Q=\sum e_{i 1}^{\imath} e_{1 i}^{i}=\sum e_{i 1}^{\imath}=\left(\sum e_{i i}\right)^{\imath}=[1]$. Hence $Q P=[1]$. Now we consider the automorphism $\gamma$ of $R_{n}$, defined 
by

$$
X^{\gamma}=Q X^{\imath} P=\sum e_{i 1} e_{1 i}^{\imath} X^{\imath} e_{j 1}^{\imath} e_{i j} .
$$

Then $e_{i j}^{\gamma}=e_{i 1} e_{11}^{i} e_{1 j}=e_{i 1} e_{1 j}=e_{i j}$ for any $i, j=1,2, \cdots, n$. Now for any $[a],[a]^{\gamma}$ commutes with all $e_{i j}^{\gamma}=e_{i j}$. Hence $[a]^{\gamma}=[b]$ for some $b \in R$. It is easily seen that $\tau$ induces an automorphism of the ring of all matrices of the form $[a]$. Therefore, there exists an automorphism $\gamma^{\prime}$ of $R$ such that $[a]^{\gamma}=\left[a^{\gamma^{\prime}}\right]$ for all $a \in R$. Then for any $\left(a_{i j}\right) \in R_{n}$ we have $\left(a_{i j}\right)^{\gamma}=\left(a_{i j}^{\gamma \prime}\right)$, and $\left(a_{i j}\right)^{\prime}=P\left(a_{i j}^{\gamma \prime}\right) Q$. Therefore, from $\alpha=\iota \eta$, we have $\left(a_{i j}\right)^{\alpha}=\left(a_{i j}\right){ }^{\imath \eta}=P^{\eta}\left(a_{i j}^{\gamma \prime}\right)^{\eta} Q^{\eta}=P^{\eta} W\left(a_{i j}^{\gamma / \sigma}\right) V Q=U^{-1}\left(a_{i j}^{\alpha \prime}\right) U$ where we set $U=V Q^{\eta}, \alpha^{\prime}=\gamma^{\prime} \sigma^{\prime}$. Thus Theorem 3 is proved.

From Baer's Theorem [3, p. 39] we know that the ring $I$ of all integers satisfies the above condition (i) for all $n$. Since $I$ can be imbedded in a field, (ii) is also satisfied for all $n$. From Theorem 3, therefore, it follows that any automorphism of the full matrix ring $I_{n}$ is inner.

The author wishes to express his gratitude to Professor S. A. Jennings for help and encouragement in the preparation of this work.

\section{REFERENCES}

1. R. Baer, Linear algebra and projective geometry, Academic Press, 1952.

2. - A unified theory of projective spaces and finite abelian groups, Trans. Amer. Math. Soc. vol. 52 (1942) pp. 283-343.

3. - The significance of the system of subgroups for the structure of the group, Amer. J. Math. vol. 61 (1939) pp. 1-44.

4. - Automorphism rings of primary abelian operator groups, Ann. of Math. vol. 44 (1943) pp. 192-226.

The University of British Columbia 